\title{
Cue-Centered Therapy for Youth Experiencing Posttraumatic Symptoms
}

Hilit Kletter, PhD*®

Ryan Matlow, PhD

Selma Tanovic, MD

Victor Carrion, MD

\author{
Address \\ "Department of Child/Adolescent Psychiatry, Stanford University School of Med- \\ icine, 401 Quarry Road, Stanford, CA, 94305, USA \\ Email: hkletter@stanford.edu \\ Published online: 19 April 2021 \\ (C) The Author(s), under exclusive licence to Springer Nature Switzerland AG 2021
}

This article is part of the Topical Collection on Early Life Trauma

Keywords Pediatric posttraumatic stress disorder - Complex trauma - Allostatic load · Psychotherapy · Manual-based intervention

\begin{abstract}
Purpose Few of the existing evidence-based interventions for child trauma exposure were specifically designed to address experiences and outcomes of complex developmental trauma. Stanford's cue-centered therapy (CCT) was designed to address this gap by offering a flexible, integrative, and insight-oriented treatment approach that is grounded in principles of neuroscience, developmental trauma, client empowerment, and allostasis. This article reviews the CCT rationale, treatment components, evidence base, and training approach.

Recent findings Studies demonstrate promising outcomes indicating CCT effectiveness in reducing child and caregiver posttraumatic stress, and in improving child functioning. Further research, however, is needed to identify which clients are best-suited for CCT (versus other available child trauma treatments) and to identify which components of CCT are most critical for addressing complex developmental trauma.

Summary CCT advances the field of child trauma treatment by offering an intervention approach focused on addressing complex developmental trauma. Positive treatment and training outcomes indicate utility of CCT for clients and clinicians. Innovations in research and training approaches are needed to further dissemination and implementation of CCT and other related child trauma interventions for complex developmental trauma.
\end{abstract}




\section{Chronic trauma exposure in children}

A large number of youths are exposed to traumatic events in their lifetime. Surveys on nationally representative community samples demonstrated that the prevalence of sexual assault was $8-10 \%$ and physical assault $17-70 \%$ in youth. Thirty-eight percent of youth had witnessed serious community violence, and $18 \%$ had lost a friend or a family member due to homicide, with high rates $(20 \%)$ of those experiencing multiple traumatic events [1]. Most youths successfully recover after a single-event trauma exposure [2]. In some children and adolescents, however, trauma exposure may lead to development of posttraumatic stress symptoms that can be heterogenous and depend on the child's age, type of trauma, and duration of exposure $[2,3 \bullet]$. In many cases, children's responses to trauma can entail classical symptoms of posttraumatic stress disorder (PTSD) including re-experiencing, avoidance, negative alterations in cognition and mood, and alterations in arousal and reactivity [4]. However, many children, especially those with chronic trauma, might exhibit additional, alternative, or overlapping symptoms of anxiety, depression, attention-deficit hyperactivity disorder, suicidal ideation, dissociative disorders, and substance abuse $[2,5 \bullet$, 6-7].

It is crucial to distinguish between type I (single event) and type II (multiple event) trauma as their phenomenology and impact differ significantly [8]. Chronic exposure to stress and adversity causes the hypothalamic-pituitary-adrenal (HPA) axis to adapt in a way that it becomes over-sensitized, and thus continuously activated, leading to potentially lifelong alterations in neurobiological regulatory systems, with longterm consequences on physical and mental health [5•, 9]. If left untreated, cumulative exposure to trauma in childhood also significantly impacts adult mental health and overall functioning, even after adjusting for trauma risk factors and developmental context [10]. Multiple traumatic events and in particular repeated interpersonal trauma pervasively affect social-emotional development; attachment and relationship formation; emotional, behavioral, and physiological regulation; cognition (executive functions, memory, and attention); personality development; and perception of self $[5 \bullet, 8,11,12]$. "Complex developmental trauma" is a term developed by researchers to capture both the exposure to childhood multiple or chronic traumatic experiences as well as the subsequent diverse range of effects on psychosocial functioning and neurodevelopment $[12,13]$. It requires interventions specifically designed to target dysregulation in above mentioned domains of functioning [11].

\section{Best practices and evidence-based psychosocial inter- ventions for complex trauma}

The International Society for Traumatic Stress Studies (ISTSS) Expert Consensus Guidelines for treatment of complex trauma emphasize the importance of adequate symptom recognition as well as employment of multimodal interventions organized in three consecutive phases focusing on (1) safety, education, and coping skills; (2) work on traumatic memories (narration and processing); and (3) treatment integration and consolidation $[14 \bullet]$.

Current best practices for children and adolescents with trauma entail sequential multi-component treatments that encompass the following elements: administering trauma-informed child and caregiver assessment; conducting crisis management with focus on stabilization and safety; encouraging development of competence, communication skills, and coping strategies; establishing strong alliance with both the child and caregiver; educating about stress and trauma responses; facilitating the process of emotion labeling, expression, and regulation; working on trauma exposure and processing (through narration or otherwise); and encouraging mind-body integration and consolidation of learned skills, as well as working on self-perception, self-efficacy, and empowerment $[15,16 \bullet]$. Detailed review of psychosocial treatments for complex trauma is beyond the scope of this article, but we will mention some of the interventions that have been empirically examined in randomized controlled trials (RCTs).

Trauma-Focused Cognitive Behavioral Therapy (TFCBT) is based on eight PRACTICE components, psychoeducation, parenting skills, relaxation skills, affective modulation skills, cognitive coping skills, trauma narrative and cognitive processing of the trauma narrative, in vivo mastery of trauma reminders, conjoint childparent sessions, and enhancing safety and future development, that are organized in three phases with a strong emphasis on a secure therapist-client relationship [17]. It has been researched in single-event and complex trauma contexts and has been adapted for several special populations $[18,19]$. 
Attachment, self-regulation, and competency (ARC) training is a modularized intervention approach that includes the core elements of trauma treatment but is framed around the developmental impact of childhood trauma with an emphasis on caregiverchild relationship dynamics (Blaustein \& Kinniburgh, 2018 ). As its foundation, ARC emphasizes attachment-focused interventions with caregivers that include caregiver affect management, caregiver-child attunement, consistent caregiver responses, and household routines and rituals. ARC builds on the attachment foundation to then address child selfregulation and self-expression skills, competency development, and trauma experience integration.

Eye movement desensitization and reprocessing (EMDR) therapy is a trauma-focused treatment first developed for adults and later adapted for children [20, 21]. The approach is based on the adaptive information processing model (AIP) that reflects complex interplay between maturation of neuronal memory systems, attachment formation, and development of self. This interaction might be interrupted by early repeated traumatic events, which leads to dysfunctional memory formation and storage, and manifests with symptoms of complex trauma [21]. EMDR utilizes techniques to access, reprocess, and integrate these maladaptively encoded memories [22].

Child-parent psychotherapy (CPP) is an empirically evaluated, psychoanalytically informed dyadic relationship-based intervention targeting early social and emotional aspects of developmental trauma. It also uses elements from cognitive behavioral therapy and play therapy to focus on attachment strengthening and partnership building between young children (age 0-5 years) and their parents who have been exposed to violence [23-25].

One of the common ways for young children to express trauma symptoms or reenact trauma is through posttraumatic play that is oftentimes repetitive and nonimaginative. Play therapies such as trauma-focused integrative play therapy (TFIPT) for sexually abused children allow children to process and express their trauma narrative in a non-verbal way with the help of different techniques of play and art [26].

School-based CBT-based group interventions, such as Cognitive-Behavioral Intervention for Trauma in Schools (C-BITS), are cost-effective, easy to administer, and particularly suitable for delivering therapy in cases of mass trauma, community violence, natural disasters, or in underserved populations when individual psychotherapy is not readily accessible $[27,28]$. Bounce back is an intervention for elementary school students with trauma that integrates elements of TF-CBT and C-BITS that has demonstrated promising results in a clinical trial [29].

Three meta-analyses evaluating RCTs of trauma interventions demonstrated efficacy of psychosocial treatments in reduction of pediatric PTSD symptoms $[30 \bullet ; 31 \bullet, 32]$. The interventions' effectiveness was confirmed when treating children exposed to multiple traumatic events [30]. CBT-based treatments were the most empirically supported and were proven to be superior to wait list and treatments as usual, regardless of treatment modality and type of participation (child only versus child and parent) $[31 \bullet, 32]$. Among the evaluated treatments, TF-CBT was the most extensively studied intervention with the largest therapeutic effects for posttraumatic symptoms $[30 \bullet, 31 \bullet]$.

\section{Psychopharmacology}

Research on the efficacy of psychopharmacology treatment in children is limited, and psychosocial treatments remain the mainstay of therapy. In the metaanalysis performed by Morina et al. (2016), out of 41 RCTs analyzed, only two assessed efficacies of medications in pediatric PTSD, and both were performed on small and highly heterogenous samples [30•]. If used, pharmacotherapy is the most beneficial when in conjunction with psychosocial interventions. When choosing the right therapeutic approach, it is important to consider the child's age, type and onset of trauma, intensity of symptoms, functional impairment, comorbid conditions, and accessibility of evidence-based psychotherapies [33, 34]. The first line of pharmacological treatment is selective serotonin reuptake inhibitors (SSRIs) as they are effective in both PTSD symptoms and concomitant panic disorder, anxiety, and depression, followed by serotonin norepinephrine reuptake inhibitors (SNRIs), such as venlafaxine and duloxetine. They are generally well tolerated and safe to use in pediatric populations but might increase suicidality, which warrants safety monitoring, especially at the beginning of treatment. Other serotoninergic medications, such as tricyclic antidepressants, are used less commonly in children. Psychotic symptoms, severe mood alterations, or aggressive behavior can be targeted with second-generation antipsychotics (risperidone, aripiprazole, quetiapine). Children with overlapping attention-deficit hyperactivity disorder can benefit from stimulants, alpha agonists, and bupropion. Propranolol and alpha agonists 
(clonidine, guanfacine) decrease sympathetic stimulation and are effective in controlling hyperarousal and re-experiencing, which might promote effectiveness of psychosocial therapy [35-37].

\section{Rationale for development of cue-centered therapy}

Due to the heterogeneity and idiosyncrasy of symptoms, complex developmental trauma, with its allpervasive emotional, relational, cognitive, behavioral, and physiological impacts, remains diagnostically and therapeutically challenging. More research is needed to investigate novel ways to approach its diverse manifestations, and new interventions need to be developed to better help, equip, and empower children who are facing ongoing stressors in life. While existing trauma treatments for children have demonstrated effectiveness in reducing complex traumatic stress, few were specifically or explicitly designed or structured to address childhood experiences of complex or chronic trauma. Therapists and mental health providers often struggle with how to comprehensively integrate and address histories of complex, chronic, or ongoing trauma exposure, and there remains a need for trauma therapies to apply concepts and practices most relevant to complex developmental trauma. In particular, targeting the development of youth insight regarding the pervasive and cumulative impact of traumatization within a broader context of adversity is particularly warranted in contexts where caregiver availability may be limited (thereby limiting opportunities to directly address relationship dynamics). Furthermore, strengths-based approaches for complex developmental trauma that present specific opportunities to identify child resilience, incorporate existing child skills, and promote child agency and empowerment may increase treatment engagement, retention, and generalized application of skills. Additional research is needed to determine whether interventions whose framing, conceptualization, and structure are geared to address complex developmental trauma (or type II trauma) lead to different or improved outcomes relative to interventions designed to address type I traumas. Given the heterogeneity of complex trauma presentations, specific components or elements of trauma-focused interventions may be particularly well-suited for certain children, depending on trauma history characteristics and other contextual factors; however, in the case of complex developmental trauma, we have yet to identify which treatments (and which treatment components) work best for whom.
Furthermore, training for clinicians and practitioners that is specifically focused on the concepts and best practices relevant to complex developmental trauma may improve provider effectiveness in both selecting and delivering trauma interventions as they encounter the diverse and varied presentations of child trauma exposure in clinical practice.

Stanford's cue-centered therapy (CCT) was designed to specifically address complex developmental trauma in youth ages $8-18$ with any trauma type. CCT is a hybrid model that integrates elements from insight-oriented, cognitive behavioral, narrative, exposure, psychoeducational, and family therapy approaches to target trauma-related emotions, thoughts, physiological reactions, and behaviors. Within a general overarching treatment structure and framework, CCT invites therapists to integrate specific approaches from their own practice and theoretical orientation(s) (e.g., cognitive restructuring, interpersonal processing, play therapy, mindfulness) to address children's experiences of complex developmental trauma.

The intervention emphasizes empowerment through knowledge using an insight-oriented approach that educates youth about (1) how traumatic cues develop through conditioning and (2) how these cues impact emotions, cognitions, physiology, and behaviors. Increased insight regarding conditioning processes provides children with an opportunity to develop new reactions and response patterns to trauma cues, thereby increasing flexibility and agency while not undermining protective survival responses in the face of potential ongoing threat or danger. CCT includes explicit psychoeducation and visual icons that emphasize the four primary domains of the trauma response (i.e., emotions, cognitions, physiology, and behaviors), leading to trauma processing that links trauma cues to response patterns across the four domains. In this, the CBT triangle is expanded to also include physiological reactions as a means of developing interoception skills that are particularly relevant for many traumatized youth. Furthermore, CCT is unique in its focus on the accumulation of traumatic stressors throughout the life span (i.e., the allostatic load), rather than anchoring treatment on an index trauma, in order to get a comprehensive picture of both youth adversity and resilience. CCT emphasis on work with the child-wherein the number of sessions requiring caregiver attendance are limited-is particularly well-suited for (1) school- and community-based 
settings, (2) developmentally older and more independent youth, and (3) contexts of ongoing family adversity that present barriers to caregiver attendance. Core CCT concepts and approaches are described below in further detail.

\section{CCT treatment model and structure}

CCT is a flexible and integrative treatment approach that incorporates the core components of trauma-focused intervention established in the current evidence base and literature $[15,38,39]$. As primary components of the therapy process, clients engaged in CCT receive psychoeducation about the impact of complex developmental trauma, develop and practice coping and self-expression skills, conduct narrative processing to integrate trauma experiences, practice gradual exposure to trauma cues, and cultivate a sense of empowerment in responding to life stressors and traumas through the therapeutic experience [40•]. At its core, CCT aims to build clients' insight about the impact of trauma in their lives across domains of cognitive, emotional, physiological, and behavioral functioning. This insight is experientially applied through narrative reflection, trauma reformulation, and exposure practice to increase clients' confidence in their ability to adaptively respond to reminders (cues) of past traumas, as well as future exposure to stress, trauma, or adversity. While the core practices and elements of CCT are consistent with established best practices in trauma treatment, CCT offers a shift in conceptualization, structure, and delivery that emphasize the sequelae of exposure to multiple or chronic traumas in childhood. CCT applies the principles of classical conditioning to the experience of complex developmental trauma as a framework for understanding the emergence of trauma-associated cued reactions (see Fig. 1). Mechanisms of cue conditioning are emphasized as primary targets throughout CCT (i.e., psychoeducation, life narratives, trauma processing, and gradual exposure). Through the course of CCT, clients identify, learn, and practice new adaptive associations between formerly neutral trauma cues (i.e., conditioned stimuli) and conditioned trauma responses as a means of increasing flexibility of response options. For example, one of the first author's clients developed a cue of loud noises (i.e., conditioned stimuli) after he witnessed a shooting (i.e., unconditioned stimuli). His sensitivity to gunshots intensified through repeated subsequent indirect exposure to violence in his community, as distant sound of guns or fireworks could be heard in his community with regularity, and he learned of numerous stories and reports of shootings resulting in injury or death. His fear
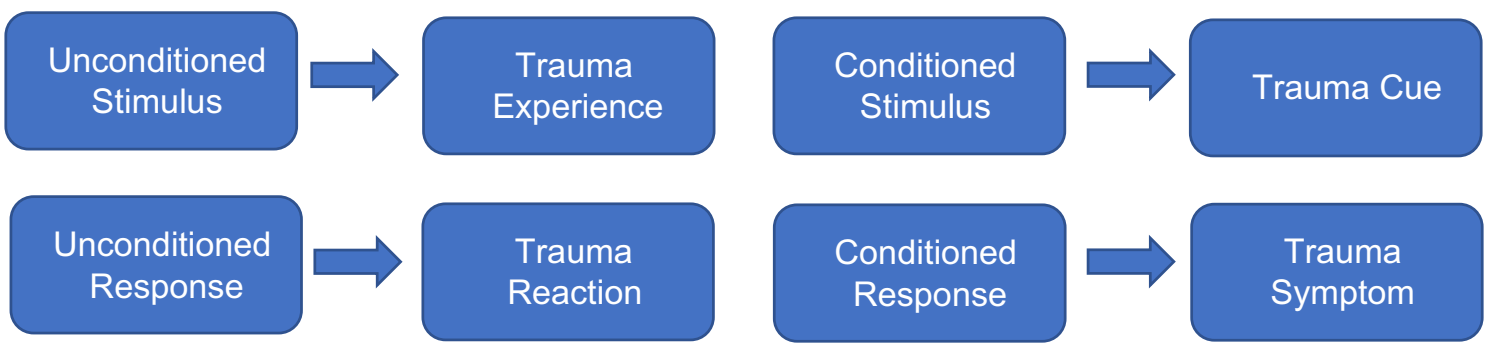

Fig. 1. CCT application of classical conditioning for trauma. 
of hearing the gunshots (i.e., unconditioned response) became associated with loud noises (e.g., a door closing with vigor, an item falling to the ground) such that any time he encountered an unexpected sound he would tense, his heart would start pounding, and he would worry that another shooting was happening (i.e., conditioned response). During CCT, he was gradually exposed to imagining loud noises, then listening to audio of loud noises, and finally having his family make loud noises at random times at home to practice developing new responses such as progressive muscle relaxation and deep breathing to override his conditioned trauma response. While the initial shooting was addressed in the client's trauma narrative, the primary therapy target was the cued response that emerged and was reinforced across multiple events and experiences throughout the client's childhood. Of note, CCT does not aim to extinguish all conditioned trauma responses given that these reactions may have originally served an adaptive function and/or may be protective in the face of future trauma exposure (e.g., future exposure to gun violence), despite such reactions being maladaptive in some contexts (hence a focus on "flexibility" over "replacement"). A goal of CCT is to empower children to evaluate their circumstances and select the response option that is most adaptive in their current context. CCT conceptualization also entails a focus on the lifetime accumulation of stressors (as well as resources and resilience factors) that shift

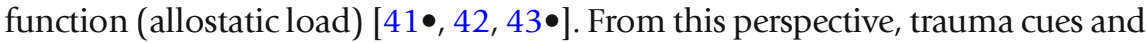
corresponding reactions may emerge and evolve over time as life experiences and stressors accumulate and interact. Further, principles of allostasis and developmental trauma theory $[44,45 \bullet]$ convey understanding that trauma reactions may present as clinically subthreshold symptoms or diffuse yet pervasive functional impairments that are not always captured or manifest in the classic symptoms of PTSD. CCT is structured to provide opportunities for children to practice and develop skills in executive functioning, self-expression, self-regulation, and interpersonal functioning while using a developmental model to conceptualize the impact of trauma exposure. Finally, CCT utilizes caregiver involvement whenever possible in order to address the vital role of caregivers in supporting and reinforcing children's adaptive responses to trauma exposure and trauma cues. However, CCT also recognizes that caregiver participation is not always available and that this should not limit therapeutic engagement with the child. In order to address this, CCT requires caregiver presence in only a few strategically placed therapy sessions in order to minimize treatment disruptions caused by barriers to caregiver attendance that can be common for children and families facing chronic or complex trauma.

CCT is classically delivered in 15 sessions that proceed through 4 treatment phases (see Table 1), though it allows for flexible augmentation to include additional sessions when clinically indicated. It is a very collaborative approach in which the youth partners as a co-investigator with the therapist throughout the intervention to build self-efficacy and empowerment. Examples of this include allowing the youth to decide which coping skills to include in their coping toolbox and which cues to work on for exposures. In addition, with therapist guidance, the youth acts as co-informant during mid-therapy to provide caregivers with an update on skills learned and treatment progress. Finally, throughout the treatment process the therapist engages the child in developing new responses to trauma reactions. 


\section{Table 1. ССT phases and components}

Phase 1: Trauma introduction and engagement

-Trauma-oriented assessment

-Psychoeducation

- Coping skill development

Phase 2: Chronic traumatic stress history

- Life timeline

- Trauma narrative

-Trauma processing

Phase 3: Cue processing and gradual exposure

- Cue identification and cue response

-Imaginal exposure to cue(s)

-In-session sensory exposure to cue(s)

-At-home in vivo exposure to cue(s)

Phase 4: Treatment integration and summary

-Re-visit trauma narrative

-Prepare for potential future challenges or exposure to adversity

- Review and celebrate progress

In phase 1, clients and therapists engage around trauma through traumafocused assessment of psychosocial history and functioning and through comprehensive psychoeducation about complex trauma. CCT psychoeducation emphasizes concepts that are most relevant for the experience of complex developmental trauma (e.g., articulating how protective reactions to trauma and adversity become conditioned during development, leading to responses and actions that are maladaptive in other contexts). Psychoeducation involves developmentally tailored learning about classical conditioning, including emphasis on the significant role of avoidance in maintaining trauma symptoms. Classical conditioning paradigms offer flexibility in conceptualizing the development of trauma responses, as conditioned responses to trauma cues may evolve due to the imprinting of a single severe trauma experience or may develop gradually over the course of repeated exposure to stress and adversity during development. Childfriendly analogies and examples are used to demonstrate the cumulative burden (and related risk) of chronic stress or multiple trauma exposure (i.e., the allostatic load). For example, CCT uses the metaphor of a backpack to represent the allostatic load and cumulative burden of trauma and adversity exposure, noting that the treatment provides an opportunity to build skills and "strength" to help the youth carry their backpack. Clients come to understand and recognize how exposure to chronic stress and trauma influences emotions, thoughts, physiological reactions, and behaviors. Phase 1 also includes the introduction and practice of coping skills that involve emotion identification and expression, relaxation, mindfulness, visual imagery, and cognitive reformulation. Clients ultimately identify and select the coping tools that are most relevant and accessible to them, including self-developed tools and practices. 
Phase 2 involves processing the client's trauma experiences and broader life history. Clients complete a life timeline to consider their multiple traumas and adversities within the context of broader life events and positive experiences. The life timeline narrative facilitates a more comprehensive organization and coherence of the youth's entire life experience that incorporates multiple complex traumas and adversities. Clients engage in the narrative re-counting of select traumas as they gradually develop awareness and understanding of the cues and trauma reactions specific to their life experiences. Life timeline and trauma narrative activities segue into a deeper processing of clients' traumatic stress histories, which involves the strategic application of phase 1 psychoeducational concepts (e.g., cue conditioning) and coping skills to clients' specific trauma experiences and reactions. Trauma processing can incorporate a range of activities from a variety of modalities including (but not limited to) cognitive restructuring, interpersonal processing, mindfulness and acceptance skills, perspective-taking, and narrative development.

In phase 3, clients identify and select the formerly neutral cues that have become associated with their trauma experiences and trauma reactions and then engage in a process of gradual exposure to increase flexibility and confidence in responding to cues. Clients process cues by identifying links to specific responses across the four domains of impact and then by selecting the adaptive response(s) to be applied in the presence of cues. This is put into practice through an exposure process that moves through imaginal exposure, insession sensory exposure, and at-home in vivo exposure to cues.

Finally, phase 4 focuses on integrating and solidifying the prior treatment activities. Clients re-visit their trauma narrative to apply and reinforce the new insight and skills developed through the course of CCT. The closing narrative provides an additional opportunity to more fully integrate experiences of trauma, adversity, recovery, and resilience following the exposure phase of treatment. Then, a termination and closing process reviews treatment progress, helps prepare for potential future trauma and stress exposure, addresses remaining challenges or needs, and celebrates client efforts. Clients finish CCT having (1) developed insight about the impact of trauma exposure (both in general and in their personal experience), (2) identified effective coping skills and practices, and (3) gained experience in adaptively approaching trauma histories and trauma cues. As a result, CCT serves to reduce client posttraumatic stress, improve functioning, increase client capacity and resources, and foster client empowerment and resilience in the face of future stressors, adversities, or traumas.

\section{CCT research}

To date, two RCTs have been done with CCT. The first involved sixty-five youth between the ages of 8-17 (average age 11.56) from 13 low-income, high risk schools in the San Francisco Bay Area, California [46]. The majority were minorities consisting of 33 African American, 26 Latino, 1 Pacific Islander, and 5 mixed. All individuals had at least two traumas with an average of five; the most common traumas were separation/loss (75\%), witnessing violence $(61.5 \%)$, homicide $(51.9 \%)$, physical abuse $(25.0 \%)$, and bullying $(25.0 \%)$. Participants were randomly assigned to CCT or a wait list control group and to 
one of two therapists. PTSD symptoms were assessed using the UCLA PTSD Reaction Index for DSM-IV child and parent report (PTSD-RI), child anxiety symptoms were assessed using the Revised Manifest Children's Anxiety Scale (RCMAS), child depression symptoms were assessed using the Children's Depression Inventory (CDI), and therapists rated overall functioning using the Children's Global Assessment Scale (CGAS). In addition, parent anxiety and depression were assessed using the Beck Anxiety Inventory (BAI) and Beck Depression Inventory (BDI). Assessments were done pre-treatment, mid-treatment, post-treatment, and at three-month follow-up. Results found reduced child symptoms of PTSD (both by child and caregiver reports), anxiety, and depression as well as overall improvement in functioning. Further, caregivers in the CCT group had greater reductions in anxiety but no significant changes in depression compared to the wait list control group. These gains were maintained at 3-month follow-up.

The second RCT included 73 youths between the ages of 7-17 (average age 12.9) a community-based mental health agency in northern California [47]. It was an ethnically diverse group consisting of 21 mixed race, 10 Latino, 12 African American, 21 Caucasian, 1 Asian, and 1 Native American. Seven participants did not report ethnicity. Participants were randomly assigned to one of three treatment conditions: cue-centered therapy (CCT), trauma-focused cognitive behavioral therapy (TF-CBT), or treatment as usual (TAU). They were also randomly assigned to a therapist in each treatment condition. Child PTSD and depression symptoms were assessed using the same measures as the first trial. Child anxiety was assessed using the Multidimensional Anxiety Scale for Children (MASC). In addition, executive functioning was assessed using the Behavioral Rating Inventory of Executive Function (BRIEF) for child report and the Brief Impairment Scale (BIS) for parent report. Assessment also included functional near-infrared spectroscopy (fNIRS) to identify neuromarkers associated with better treatment outcome. Preliminary analyses show promising results for both TF-CBT and CCT. A factorial ANOVA comparing T1 to T3 indicated a main effect of time on total scores on the PTSD-RI across CCT $(N=6, M=41.4$, SD $=21.6)$, TFCBT $(N=7, M=37.6, S D=18.5)$, and TAU $(N=5, M=44.5, S D=14.3)$ and a significant treatment $\mathrm{x}$ time interaction. Follow-up post hoc tests revealed significantly lower post-treatment scores on the PTSD-RI for the CCT $(M=$ $23.4, \mathrm{SD}=18.6, d=1.39)$ and TF-CBT $(M=18.5, \mathrm{SD}=10.65, d=1.08)$ group, but not for TAU $(M=42.17, S D=19.3)$. There were also significant main effects of time on the CDI and MASC total scores, but no significant treatment by time interactions at this time.

Recently, two studies were conducted using the data set from the first RCT. The first study examined parent-child informant discrepancy in rating of child PTSD symptoms pre- and post-treatment [48]. In contrast with prior research findings, no significant correlation was found between parent and child PTSD ratings across time points, and there was no significant change in discrepancy of reporting over the course of CCT. The difference of this finding from prior research may be explained by the chronicity of the trauma and high level of adversity experienced by the youth in this study. This suggests that when conducting assessment for complex trauma interventions such as CCT, one needs to be aware that there may be greater disagreement between the caregiver and child on child trauma symptoms. 


\section{СCT training and dissemination}

The second study examined the association between trauma narrative characteristics and symptom severity [49]. In CCT trauma narratives are obtained at two time points in treatment (T1, sessions $4-5$ and $\mathrm{T} 2$, session 13). Thus, trauma narratives were also compared at these two time points to examine changes across treatment and to determine how these changes influenced treatment outcome. Results found that overall use of positive emotional language (e.g., "I was glad") was associated with lower pre-treatment levels of depression and anxiety. In addition, overall use of temporal connectives (e.g., before, then) was associated with lower pre-treatment depression. Further, overall use of positive conceptual language (e.g., "I realized") was associated with higher levels of pre-treatment PTSD symptoms. Greater chronological organization was associated with higher pre-treatment PTSD and anxiety symptoms. Across time points, narratives did not differ significantly with the exception of a decrease in emotional language. In addition, increased use of positive conceptual language at T2 was significantly associated with decreased parent report of PTSD symptoms. Increased use of positive emotional language at T2 was associated with increases in anxiety. These results suggest that trauma narrative styles that are adaptive early on in the CCT process may not be so after youth have completed the intervention. The patterns found with emotional and conceptual language suggest that having a positive outlook may be a protective factor at the beginning of CCT; however, it may not be clinically useful for therapists to avoid disrupting a youth's unrealistically positive perspective on their trauma. In addition, high levels of cognitive language early in treatment do not necessarily imply a child that is coping well and may actually indicate an intellectualized or ruminative style. Given that interventions such as CCT contain a significant cognitive processing component, an increased focus on cognition during the narrative retelling may be an indication of treatment improvement as the child has mastered the cognitive restructuring skill.

\section{CCT training model and rationale}

Despite the increasing number of emerging psychosocial evidence-based treatments, their implementation in clinical practice still remains challenging and the research on the mechanisms of their successful dissemination is largely lacking $[50,51]$. Successful dissemination and implementation require both an increase in knowledge about the intervention and a change in providers' behavior and are determined by four factors: quality of training, institutional support (in particular quality and availability of supervision), providers' individual characteristics including theoretical orientation preferences, and clients' characteristics. A well-organized and executed training, consisting of a treatment manual, in-person workshop, and clinical supervision, can facilitate community implementation [52]. Sholomskas et al. (2005) demonstrated that training combining an intervention manual with a didactic seminar and case consultations is superior to manual review in increasing knowledge and skills in CBT interventions [50]. Case consultation is another crucial factor in learning, but the delivery and nature of clinical supervision can vary greatly between carefully crafted and controlled study protocols and oftentimes unpredictable, non-conforming, and under-resourced community settings, which must be considered when planning intervention dissemination and implementation [53]. 
Since the beginning, the CCT team has been following best practices in development of a training program for clinicians $[52,54]$ to include the CCT treatment manual, in-person training, and clinical supervision. The manual provides a theoretical framework, written instructions, as well as a large number of visuals and worksheets for clinicians to guide them through sessions and facilitate the intervention. It is available in English and Spanish [40•, 55]. The next step in training was an instructor-led in-person workshop conducted by CCT master trainers. The workshop was delivered to individuals within university and community clinical settings in groups of various sizes (ranging from 4 to 27 participants) for a duration of 2 days and with adjustments in certain cases (condensed 2-h or 4-h overviews or 1 day of training). Special care was taken to incorporate balanced amounts of didactic teaching and competence training and utilize active learning techniques such as role plays, behavior modelling, and opportunities to practice clinical skills that are known to elicit behavioral changes in providers and increase utilization of treatment $[52,56]$.

So far, 231 local clinicians (90 of them in the period of September 2019March 2020) in the San Francisco Bay Area, California, have been trained in CCT as part of clinical study protocols, through internships and fellowships for doctoral and postdoctoral students, medical residents, and fellows, and in local partner community organizations. In addition, 2347 therapists were trained in workshops as part of a disaster response program in Santa Rosa, California, and Puerto Rico. These workshops integrated CCT with preventive interventions, such as mindfulness and yoga, in collaboration with Pure Edge, Inc., an organization dedicated to the training of educators and others on mindfulness and yoga. International outreach includes specific training partnerships for students and clinicians in Spain, Israel, and Central and Latin America, as well as provision of CCT training workshops at international trauma conferences [57].

The final step in training involves supervision on a minimum of two CCT cases. Thus far, 32 trainees have received case consultation-based supervision by master trainers consisting of 15 calls for $1 \mathrm{~h}$ a week. Case consultations were conducted in accordance to best supervision practices, including providing feedback, ongoing observation, defining clear goals, employing efficient communication strategies and reflective techniques, focusing on clinical problem solving, assessing fidelity, and monitoring outcomes [58]. The extent of CCT master trainer availability and capacity to provide ongoing supervision has been a limiting factor in trainee completion of the CCT training program, highlighting the need to develop a formalized train-the-trainer program, an effort which is currently underway.

Over the last two decades, there have been initiatives to migrate manualized content of empirically supported interventions into an online format, which led to the emergence of new engaging instructional design methods, developed specifically for web-based learning [59, 60]. The benefits of an online format include greater accessibility especially for international reach, cost effectiveness, building a sense of community, greater ease in updating the content, and selfpaced learning along with the ability to review the content as much as the learner would like. There are more and more empirically supported interventions that are including web-based instruction into their training including TF- 
CBT (http://tfcbt.musc.edu/), CBT (http://www.behavioraltech.com/ol/), and DBT (https://psychwire.com/linehan). Interactive multi-media online training for DBT was empirically proven to be better for learning DBT skills in community settings compared to treatment manual and in-person training led by instructors [61].

In 2018, in an effort to enhance learning CCT skills, bring into play technological innovations, and improve accessibility, the CCT team in partnership with Educational Technology (EdTech) at the Stanford School of Medicine started developing a new sequential program that incorporates online learning into the training experience (as a second step, after the manual review, and before the in-person workshop). The CCT online course is organized in eight clinically oriented, self-paced, interactive online modules with content presented in multi-media format and created in accordance to the ADDIE instructional design model [59]. Prior to scripting and production of the online modules, learner's needs had been assessed and the potential course design discussed in focus groups and in-depth interviews with clinicians that were already trained in CCT in-person.

\section{Training evaluations and recommendation}

All levels of CCT training have been consistently informed by trainees' feedback and optimized to address the specific learning needs of mental health professionals working in a wide range of clinical settings. Trainees' evaluations were considered when planning in-person training content and activities for every consecutive workshop, which contributed to a trend in increase with overall satisfaction with the instruction over time.

In terms of online course needs assessment, focus groups and in-depth interviews included feedback that had been gathered from 11 participants in 30-60 min videoconference or telephone calls. All clinicians were positive about the development of the online course and expressed enthusiasm about it. They endorsed the proposed innovative concept based on clinical vignettes, as put by one of the participants:

... I think in general, the description of the course sounds very interesting and that it provides things that not all courses have, in terms of choosing the intervention and seeing how it plays out, so it sounds much more interactive than most courses [...] it is not standard, but goes above and beyond.

Due to the COVID-19 pandemic, however, the initial instructional design had to be modified so that production of the course is fully executed remotely with no in-person interaction. At the moment of writing this article, the prototype module is being beta tested with 44 clinicians on functionality, engagement, and content. Results will inform creation of the remaining seven modules by iterative design that will again be tested before the final version is published and available to the public audience, which can be expected to happen by the end of 2020.

There has been a continuous effort to improve CCT training, informed by research in dissemination-implementation science, technological advancements in the domain of online instruction and by the specific needs of mental health practitioners. Systematic research needs to be done to assess the level of providers' knowledge and behavioral change with the current CCT training 
model. In addition, special attention needs to be made to identify barriers to intervention and implementation, to prevent under-utilization and ensure that newly learned skills are put into practice in the clinical setting.

\section{Conclusion and future directions}

CCT is an intervention for complex developmental trauma that has proven effective in reducing PTSD symptoms along with anxiety and depression in chronically traumatized youth. In addition, although there is minimal caregiver participation in the intervention, they seem to benefit by virtue of having their child participate such that their own anxiety and depression is reduced as well. Further research is needed to determine which components of CCT are most essential, and how to decide when CCT would be the most appropriate intervention compared to other available interventions depending on youth characteristics and clinical presentation. This will help the development of algorithms of care which are crucially needed in the field. Although we have demonstrated the initial feasibility of a CCT training and dissemination model, we are currently working on defining specific criteria for training on the intervention, hours of practice required for certification, and how to assess practitioners' knowledge. In addition, we are currently developing a train-the-trainer model to increase the accessibility of CCT. Finally, future research is needed to continue to evaluate the best modality for delivery of the intervention, reducing barriers to clinician engagement and implementation of CCT, as well as providing ongoing support and resources to build a community of CCT practitioners.

\section{References and Recommended Reading}

Papers of particular interest, published recently, have been

highlighted as:

- Of importance

-. Of major importance

1. Saunders BE, Adams ZW. Epidemiology of traumatic experiences in childhood. Child Adolesc Psychiatr Clin N Am. 2014;23:167-84. https://doi.org/10.1016/j.chc. 2013.12.003.

2. Copeland WE, Keeler G, Angold E, Costello J. Traumatic events and posttraumatic stress in childhood. Arch Gen Psychiatry. 2007;64:577-84. https://doi.org/ 10.1001/archpsyc.64.5.577.

3. $\quad$ Landolf MA, Kanardy JA: Evidence-based treatments for children and adolescents. In: Schnyder, U, Cloitre $\mathrm{M}$, editors. Evidence based treatments for traumarelated psychological disorders. Cham: Springer; 2015. Provides an overview of current treatments for childhood trauma with theoretical background and case examples.

4. American Psychiatric Association: Diagnostic and statistical manual of mental disorders. $5^{\text {th }}$ ed. Washington DC: 2013.

5. $\quad$ Carrion VG, Weems CF: Neuroscience of pediatric PTSD. New York: Oxford University Press; 2017, For a thorough summary of advances in neurobiology of pediatric PTSD in areas of functioning and development.

6. DeBellis MD. Developmental traumatology: a contributory mechanism for alcohol and substance use disorders. Psychoneuroendocrinology. 2002;27:155-70. https://doi.org/10.1016/S0306-4530(01)00042-7.

7. Famularo R, Fenton T, Kinscherf R, Augustyn M. Psychiatric comorbidity in childhood posttraumatic stress disorder. Child Abuse Negl. 1996;20:953-61. https:// doi.org/10.1016/0145-2134(96)00084-1.

8. Carrion VG, Kletter H. Posttraumatic stress disorder: shifting toward a developmental framework. Child Adolesc Psychiatr Clin N Am. 2012;21:573-91. https:// doi.org/10.1016/j.chc.2012.05.004. 
9. Schonkoff JP, Garner AS. The lifelong effects of early childhood adversity and toxic stress. Pediatrics. 2012;129:e232-46. https://doi.org/10.1542/peds. 2011-2663.

10. Copeland WE, Shanahan L, Hinesley J, Chan RF, Aberg KS, Fairbank JA, et al. Association of childhood trauma exposure with adult psychiatric disorders and functional outcomes. JAMA NetwOpen. 2018;1:e184493. https://doi.org/10.1001/jamanetworkopen.2018. 4493.

11. Ford JD, Curtois CA. Defining and understanding complex traumatic stress disorders. In: Curtois CA, Ford JD, editors. Treating complex traumatic stress disorders. New York: The Guilford Press; 2009.

12. van der Kolk BA. Developmental trauma disorder: toward a rational diagnosis for children with complex trauma histories. Psychiatr Ann. 2005;35:401-8. https://doi.org/10.3928/00485713-20050501-06.

13. Lawson DM, Quinn J. Complex trauma in children and adolescents: evidence-based practice in clinical settings. J Clin Psychol. 2013;69:497-509. https://doi.org/10. 1002/jclp.21990.

14. Cloitre M, Curtois CA, Charuvastra A, Carapezza R, Stolbach BC, Green BL. Treatment of complex PTSD: results of the ISTSS expert clinician survey on best practices. J Trauma Stress. 2011;24:615-27. https:// doi.org/10.1002/jts.20For a summary of best practice guidelines for treatment of childhood complex trauma based on expert opinion.

15. Ford JD, Cloitre M. Best practices in psychotherapy for children and adolescents. In: Curtois CA, Ford JD, editors. Treating complex traumatic stress disorders. New York: The Guilford Press; 2009.

16. Matlow R: General principles of psychotherapy. In: Carrion V, editor. Assessing and treating youth exposed to traumatic stress. Washington DC: American Psychiatric Association Publishing; 2019. Provides a summary of the core components of trauma treatment and treatment challenges.

17. Cohen JA, Mannarino AP. Trauma-focused cognitive behavioural therapy for children and parents. Child Adolesc Mental Health. 2008;13:158-62. https://doi. org/10.1111/j.1475-3588.2008.00502.x.

18. Cohen JA, Mannarino AP, Kliethermes M, Murray LA. Trauma-focused CBT for youth with complex trauma. Child Abuse Negl. 2012;36:528-41. https://doi.org/ 10.1016/j.chiabu.2012.03.007.

19. Cohen JA, Mannarino EP, Deblinger E. Traumafocused CBT for children and adolescents: treatment applications. New York: The Guilford Press; 2012.

20. Shapiro F. Eye movement desensitization and reprocessing (EMDR) therapy: basic principles, protocols and procedures. New York: The Guilford Press; 2018.

21. Gomez AM. EMDR therapy and adjunct approaches with children. New York: The Springer Publishing; 2013.

22. Clarke S, Zack S. Eye movement desensitization and reprocessing and dialectical behavior therapy. In: Carrion $\mathrm{V}$, editor. Assessing and treating youth exposed to traumatic stress. Washington DC: American Psychiatric Association Publishing; 2019.

23. Lieberman AF, Van Horn P, Ippen CG. Toward evidence-based treatment: child-parent psychotherapy with preschoolers exposed to marital violence. J Am Acad Child Adolesc Psychiatry. 2005;44:1241-8. https://doi.org/10.1097/01.chi.0000181047.59702. 58.

24. Lieberman AF, Reyes V. Child-parent psychotherapy and traumatic exposure to violence. Zero to Three. 2012;32:20-5.

25. Lieberman AF, Van Horn P. Psychotherapy with infants and young children: repairing the effects of stress and trauma on early attachment. New York: The Guilford Press; 2008.

26. Gil E. Trauma-focused integrated play therapy (TFIPT). In: Goodyear-Brown P, editor. Handbook of child sexual abuse: identification, assessment and treatment. Hoboken: John Wiley \& Sons, Inc.; 2011.

27. Jaycox LH, Kataoka SH, Stein BD, Langley AK, Wong M. Cognitive behavioral intervention for trauma in schools. J Appl Sch Psychol. 2012;28:239-55. https:// doi.org/10.1080/15377903.2012.695766.

28. Jaycox LH, Stein BD, Wong M. School intervention related to school and community violence. Child Adolesc Psychiatr Clin N Am. 2014;23:281-93. https:// doi.org/10.1016/j.chc.2013.12.005.

29. Langley AK, Gonzales A, Sugar CA, Solis D, Jaycox L. Bounce back: effectiveness of an elementary schoolbased intervention for multicultural children exposed to traumatic events. J Consult Clin Psychol. 2015;83:853-65.

30. Morina N, Koerssen R, Pollet TV. Interventions for children and adolescents with posttraumatic stress disorder: a meta-analysis of comparative outcome studies. Clin Psychol Rev. 2016;47:41-54. https://doi. org/10.1016/j.cpr.2016.05.006Examines the efficacy of both psychosocial and pharmacological interventions for childhood trauma.

31. Dorsey S, McLaughlin KA, Kerns SEU, Harrison JP, Lambert HK, Briggs EC, et al. Evidence base update for psychosocial treatments for children and adolescents exposed to traumatic events. J Clin Child Adolesc Psychol. 2017;46:303-30. https://doi.org/10.1080/ 15374416.2016.1220309Provides a good review of studies conducted to examine available treatment interventions for childhood trauma and advances in the evidence base.

32. Silverman WK, Oritz CD, Viswesvaran C, Burns BJ, Kolko DJ, Putnam FW, et al. Evidence-based psychosocial treatments for children and adolescents exposed to traumatic events. J Clin Child Adolesc Psychol. 2008;37:156-83. https://doi.org/10.1080/ 15374410701818293.

33. Keeshin BR, Strawn JR. Psychological and pharmacologic treatment of youth with posttraumatic stress disorder. Child Adolesc Psychiatr Clin N Am. 2014;23:399-411. https://doi.org/10.1016/j.chc.2013. 12.002 . 
34. Stamatakos M, Campo JV. Psychopharmacologic treatment of traumatized youth. Curr Opin Pediatr. 2010;22:599-604. https://doi.org/10.1097/MOP. 0b013e32833e26d5.

35. Donnelly CL. Pharmacologic treatment approaches for children and adolescents with posttraumatic stress disorder. Child Adolesc Psychiatr Clin N Am. 2003;12:251-69. https://doi.org/10.1016/s10564993(02)00102-5.

36. Donnelly CL. Psychopharmacotherapy for children and adolescents. In: Foa EB, Keane TM, Friedman MJ, Cohen JA, editors. Effective treatments for PTSD. Practice Guidelines from the International Society for Traumatic Stress Studies. New York: The Guilford Press; 2009.

37. Donnelly CL, Lubit R, Bami A. Psychopharmacology. In: Carrion V, editor. Assessing and treating youth exposed to traumatic stress. Washington DC: American Psychiatric Association Publishing; 2019.

38. National Child Traumatic Stress Network (web). Trauma treatments overview. n.d. https://www.nctsn.org/ treatments-and-practices/trauma-treatments/overview.

39. Abuse S, Administration MHS. Trauma-informed care in behavioral health services. Treatment improvement protocol (TIP) series 57. HHS Publication No. (SMA) 13-4801. Substance Abuse and Mental Health Services Administration: Rockville, MD; 2014.

40. Carrion, VG: Cue-centered therapy for youth experiencing posttraumatic symptoms: a structured multimodal intervention, therapist guide. New York: Oxford University Press; 2016. For the theoretical rationale behind CCT, guidelines for therapists practicing CCT, session-by-session goals, and session worksheets.

41. Karatsoreos IN, McEwen BS. Psychobiological allostasis: resistance, resilience, and vulnerability. Trends Cogn Sci 2011:15:576-84. Doi: 10.1016/ j.tics.2011.10.005. Provides a definition of the concept of allostatic load and reviews factors that modulate resilience.

42. McEwen BS. Stress, adaptation and disease. Allostasis and allostatic load. Ann N Y Acad Sci. 1998;840:33-44 https://doi.org/10.1111/j.1749-6632.1998.tb09546.x.

43. McEwen BS. Allostasis and allostatic load: implications for neuropsychopharmacology. Neuropsychopharmacology. 2000;22:108-24. https://doi.org/10.1016/ S0893-133X(99)00129-3Also provides a definition of allostatic load, how it's measured, and how it's applied to the field of trauma.

44. Perry BD, Hambrick EP. The neurosequential model of therapeutics. Reclaiming children and youth. 2008; 17:38.

45. $\quad$ van der Kolk BA. Developmental trauma disorder: toward a rational diagnosis for children with complex trauma histories. Psychiatr Ann. 2017;35:401-8. https://doi.org/10.13109/prkk.2009.58.8.572For an explanation of the concept of complex trauma in children and symptom presentation.
46. Carrion VG, Kletter H, Weems CF, Berry RR, Rettger JP. Cue-centered treatment for youth exposed to interpersonal violence: a randomized controlled trial. J Trauma Stress. 2013;26:654-62. https://doi.org/10.1002/jts. 21870.

47. Carrion, V, Cohen J, Lieberman A, Amaya-Jackson L. New developments in evidence-based trauma treatments and relevance for child and adolescent psychiatrists. Symposium presented at the $67^{\text {th }}$ annual conference of the American Academy of Child and Adolescent Psychiatry: San Francisco, CA; 2020

48. Friedman MA, Kletter H. Parent-child informant discrepancy and treatment response among youth exposed to chronic adversity: implications for childhood PTSD assessment and intervention. Poster presented at the $35^{\text {th }}$ annual conference of the International Society for Traumatic Stress Studies: Boston, MA; 2019.

49. Acker K. Reichert (in progress). Response to treatment as predicted by changes in the structure and content of children's trauma narratives: a mixed-method analysis.

50. Sholomskas DE, Syracuse-Siewert G, Rounsaville BJ, Ball SA, Nuro KF, Carroll KM. We don't train in vain: a dissemination trial of three strategies of training clinicians in cognitive-behavioral therapy. J Consult Clin Psychol. 2005;73:106-15. https://doi.org/10.1037/ 0022-006X.73.1.106.

51. Becker KD, Stirman SW. The science of training in evidence-based treatments in the context of implementation programs: current status and prospects for future. Admin Pol Ment Health. 2011;38:217-22. https://doi.org/10.1007/s10488-011-0361-0.

52. Beidas RS, Kendall PC. Training therapists in evidencebased practice: a critical review of studies from a systems-contextual perspective. Clin Psychol Sci Pract. 2010;17:1-30. https://doi.org/10.1111/j.1468-2850. 2009.01187.x.

53. Baerman SK, Weisz JR, Chorpita BF, Hoagwood K, Ward A, Ugueto AM, et al. More practice, less preach? The role of supervision and therapist characteristics in EBT implementation. Admin Pol Ment Health. 2013;40:518-29. https://doi.org/10.1007/s10488013-0485-5.

54. Herschell AD, Kolko DJ, Baumann BL, Davis AC. The role of therapist training in the implementation of psychosocial treatments: a review and critique with recommendations. Clin Psychol Rev. 2010;30:448-66. https://doi.org/10.1016/j.cpr.2010.02.005.

55. Carrion VG. Terapia de claves traumáticas: manual de intervención para niños y adolescentes con síntomas postraumáticos. Editorial Gedisa: Barcelona; 2018.

56. Nakamura BJ, Selbo-Bruns A, Okamura K, Chang J, Slavin L, Shimabukuro S. Developing a systemic evaluation approach for training programs within a trainthe-trainer model for youth cognitive behavior therapy. Behav Res Ther. 2014;54:10-9. https://doi.org/10. 1016/j.brat.2013.12.001.

57. Kletter, H, Matlow, R, Carrion, V. Cue-centered therapy for chronic trauma. Pre-meeting institute presented at 
the $35^{\text {th }}$ annual conference of the International Society for Traumatic Stress Studies. Boston, MA; 2019.

58. Milne D, Reiser R, Aylott H, Dunkerley C, Fitzpatrick H, Wharton S. The systematic review as an empirical approach to improving CBT supervision. Int J Cogn Ther. 2010;3:278-94. https://doi.org/10.1521/ijct.2010.3.3. 278.

59. Weingardt KR. The role of instructional design and technology in the dissemination of empirically supported, manual-based therapies. Clin Psychol Sci Pract. 2004;11:313-31. https://doi.org/10.1093/clipsy/ bph087.

60. Weingardt KR, Cucciare MA, Bellotti C, Pin LW. A randomized trial comparing two models of web-based training in cognitive-behavioral therapy for substance abuse counselors. J Subst Abus Treat. 2009;37:219-27. https://doi.org/10.1016/j.jsat.2009.01.002.
61. Dimeff LA, Koerner K, Woodcock EA, Beadnell B, Brown MZ, Skutch JM, et al. Which training method works best? A randomized controlled trial comparing three methods of training clinicians in dialectical behavior therapy skills. Behav Res Ther. 2009;47:921-30. https://doi.org/10.1016/j.brat.2009.07.011.

\section{Publisher's Note}

Springer Nature remains neutral with regard to jurisdictional claims in published maps and institutional affiliations. 